\title{
TECNOLOGIA ASSISTIVA SOBRE AMAMENTAÇÃO PARA PESSOAS COM DEFICIÊNCIA VISUAL: COMPARAÇÃO BRASIL E PORTUGAL
}

\author{
Paula Marciana Pinheiro de Oliveira1, Lorita Marlena Freitag Pagliuca², Paulo César de Almeida3, Monaliza \\ Ribeiro Mariano ${ }^{4}$, António Luís Rodrigues Faria de Carvalho ${ }^{5}$, Gisele Mendes da Silva ${ }^{6}$
}

\footnotetext{
${ }^{1}$ Doutora em Enfermagem. Professora do Instituto de Ciências da Saúde da Universidade da Integração Internacional da Lusofonia Afro-Brasileira (Unilab). Acarape, Ceará, Brasil. E-mail: paulapinheiro@unilab.edu.br

${ }^{2}$ Doutora em Enfermagem. Professora do Programa de Pós-Graduação de Enfermagem Universidade Federal do Ceará. Fortaleza, Ceará, Brasil. E-mail: pagliuca@ufc.br

${ }^{3}$ Doutor em Saúde Pública. Professor Colaborador do Curso de Mestrado Profissional em Avaliação de Políticas Públicas da Universidade Estadual do Ceará. Fortaleza, Ceará, Brasil. E-mail: pc2015almeida@gmail.com

${ }^{4}$ Doutora em Enfermagem. Professora do Instituto de Ciências da Saúde da UNILAB. Acarape, Ceará, Brasil. E-mail: monalizamariano@unilab.edu.br

${ }^{5}$ Doutor em Educação. Professor da Escola Superior de Enfermagem do Porto. Porto, Portugal. E-mail: luiscarvalho@esenf.pt

${ }^{6}$ Graduanda em Enfermagem, UNILAB. Acarape, Ceará, Brasil. E-mail: giselems@aluno.unilab.edu.br
}

\section{RESUMO}

Objetivo: descrever o resultado da validação aparente e de conteúdo da tecnologia assistiva "Amamentação em ação", junto a pessoas com deficiência visual de duas realidades, de Portugal e Brasil.

Método: estudo de validação aparente fundamentado no modelo da psicometria composto dos polos teórico, empírico e analítico. Participaram, no Brasil, 19 pessoas com deficiência visual e em Portugal 21. O construto é a Tecnologia Assistiva em formato cordel, "Amamentação em Ação", elaborada no Brasil e adaptada para aplicação também em Portugal. Para coleta, foi utilizado computador com sintetizador de voz e sistema de leitura de tela do computador para cegos. Para os itens de avaliação utilizou-se escala tipo Likert.

Resultados: entre os participantes da América do Sul prevaleceram adultos jovens com escolaridade correspondente ao ensino fundamental e entre os europeus, idosos com ensino fundamental. Concernente à tecnologia propriamente dita, em todos os tópicos e itens houve excelentes avaliações com alguns tópicos analisados diferentemente nos dois países. Os participantes gostaram da Tecnologia Assistiva, mas para validar utilizando o sintetizador, precisou-se de tempo, e mesmo assim, muitas vezes, foi complexo. Alguns se recusaram a responder isto pode estar relacionado ainda à ausência da inclusão digital.

Conclusão: concluiu-se que a tecnologia foi avaliada. A mesma foi bem-aceita e conclui-se que para pessoas com deficiência visual ainda são necessárias novas estratégias de inclusão, tanto na saúde como em outras áreas.

DESCRITORES: Aleitamento materno. Tecnologia. Pessoas com deficiência visual. Enfermagem. Promoção da saúde.

\section{BREASTFEEDING ASSISTIVE TECHNOLOGY FOR PEOPLE WITH VISUAL DEFICIENCIES: COMPARISON BETWEEN BRAZIL AND PORTUGAL}

\begin{abstract}
Objective: to describe the result of the apparent validation and content of the assistive technology "Breastfeeding in action", with visually impaired people from two realities, Portugal and Brazil.

Method: an apparent validation study based on the psychometric model composed of the theoretical, empirical and analytical poles. Participants were 19 people In Brazil and 21 people in Portugal with visual impairments. The construct is the Assistive Technology in a cordel format, "Breastfeeding in Action", developed in Brazil and adapted for application in Portugal. For data collection, a computer with a voice synthesizer and computer screen reading system for the blind was used. For the evaluation items a Likert-type scale was used.

Results: among the participants of South America, young adults with elementary education and among the Europeans, older adults with elementary education prevailed. Regarding the technology itself, in all topics and items there were excellent evaluations with some topics analyzed differently in the two countries. The participants liked the Assistive Technology, however, it took time for the validation using the synthesizer and, even then, it was often complex. The refusals to participate may have been related to the absence of digital inclusion.

Conclusion: it was concluded that the technology was evaluated. It was well accepted and it was found that, for people with visual impairment, new inclusion strategies are still necessary, both in health and in other areas.
\end{abstract}

DESCRIPTORS: Breastfeeding. Technology. People with visual impairment. Nursing. Health Promotion. 


\title{
TECNOLOGÍA DE ASISTENCIA SOBRE AMAMANTAMIENTO PARA PERSONAS CON DEFICIENCIA VISUAL: COMPARACIÓN ENTRE BRASIL Y PORTUGAL
}

\begin{abstract}
RESUMEN
Objetivo: describir el resultado de la validación aparente y del contenido de la tecnología de asistencia "Amamantamiento en acción", junto a personas con deficiencia visual de las realidades en Portugal y Brasil.

Método: estudio de validación aparente fundamentado en el modelo de la sicometría y compuesto de polos teóricos, empíricos y analíticos. En Brasil, participaron 19 personas con deficiencia visual y en Portugal 21 personas. El constructo es la Tecnología de Asistencia en el formato cordel, "Amamantamiento en Acción", elaborada en Brasil y también adaptada para su aplicación en Portugal. Para la obtención de datos se usó un computador con sintetizador de voz y sistema de lectura de pantalla del computador para ciegos. Para los ítems de evaluación se utilizó la escala de tipo Likert.

Resultados: entre los participantes de América del Sur prevalecieron los adultos jóvenes con escolaridad correspondiente a la enseñanza primaria y entre los europeos, los ancianos con enseñanza primaria. En relación a la tecnología propiamente dicha, en todos los tópicos e ítems hubo excelentes evaluaciones con algunos tópicos analizados de forma diferente en ambos países. Los participantes gustaron de la Tecnología de Asistencia, pero para validar utilizando el sintetizador se precisó de tiempo, y aun así, muchas veces resultó ser complejo. Algunos se negaron a responder por estar relacionado con la ausencia de la inclusión digital.

Conclusión: se concluyó que la tecnología fue evaluada. La misma fue bien aceptada y se concluye que para las personas con deficiencia visual todavía son necesarias nuevas estrategias de inclusión tanto en la salud como en otras áreas.
\end{abstract}

DESCRIPTORES: Amamantamiento materno. Tecnología. Personas con deficiencia visual. Enfermería. Promoción de la salud.

\section{INTRODUÇÃO}

Promoção da saúde é estratégia de mudança dos modelos técnicos e assistenciais na busca de elementos e possibilidades, saberes e fazeres, que auxiliem na qualidade de saúde e de vida da população. ${ }^{1}$ Seu conceito amplo permite interferir na vida do indivíduo de forma positiva e abrangente, incluindo os indivíduos sem exceção. Neste âmbito, deve-se priorizar o foco nas pessoas com deficiência e necessidades específicas desse público.

Com relação à deficiência visual, a Organização Mundial de Saúde (OMS) apresenta estimativa de 285 milhões de pessoas em todo o mundo, sendo que 39 milhões são cegos e 246 têm baixa visão. ${ }^{2}$

No Brasil, conforme dados do IBGE (Instituto Brasileiro de Geografia e Estatísticas) dos 23,9\% (45,6 milhões de pessoas) declararam alguma deficiência, destas, a visual perfaz um total de $3,5 \%$ da população, sendo $(16,0 \%)$ homens e mulheres $(21,4 \%){ }^{3} \mathrm{Em}$ Portugal, representam $9,16 \%$ da população, assim há 163.515 indivíduos com esta deficiência. ${ }^{4}$

Promoção da saúde deve ser proporcionada a todos, inclusive pessoas com deficiência visual. A comunicação para estas pessoas ocorre basicamente pelos sentidos remanescentes, tato e audição, por isso, os materiais preparados para eles devem possuir características específicas. ${ }^{5}$ Além disso, materiais adaptados para pessoas com deficiência visual são escassos, pois a educação em saúde ainda está voltada para pessoas sem a deficiência, utilizando manuais, panfletos e tecnologias visíveis.

Desse modo, torna-se imprescindível lançar mão da tecnologia assistiva, entendida como recursos, instrumentos, serviços que podem ser utilizados por e para as pessoas com deficiência e idosos para aumentar sua autonomia e independência. ${ }^{6} \mathrm{O}$ cego utiliza o computador com auxílio de leitor de tela que transforma o texto escrito em áudio. Para o cego, ao se elaborar tecnologia assistiva, deve ser observado que o texto esteja redigido de forma clara e objetiva; caso apresente figura, esta deve vir acompanhada de áudio descrição e, sempre que possível reproduzida em relevo para que possa ser explorada pelo tato.

Em estudo anterior, foi redigido texto denominado "Amamentação em ação" abordando composição do leite materno; mitos e tabus sobre amamentação; profissional, família e amamentação; vantagens da amamentação para a criança; vantagens da amamentação para a mãe e a família; e comunicação em saúde. Este texto foi transformado em versos segundo normas da literatura de cordel, versos rimados são atrativos, despertam e prendem a atenção do ouvinte, principal forma do cego se relacionar com o meio. Neste cenário, a literatura de cordel foi utilizada como tecnologia assistiva, disponibilizada em meio eletrônico para ser acessada no computador, constituindo-se em material de educação e promoção da saúde. Esta tecnologia assistiva foi avaliada por pessoas cegas em pesquisa realizada no Brasil.7

Construção de tecnologia assistiva deve ser submetida a processo de validação a fim de afirmar que se constitui em meio eficaz e efetivo para aprendizagem, autonomia e independência dos indivíduos. Este processo de validação deve ser conduzido em diferentes contextos culturais. Nesta perspectiva, uma das pesquisadoras, durante estágio de doutorado sanduíche, replicou o processo 
em amostra de pessoas cegas de Portugal, país que, embora com o mesmo idioma, apresentam peculiaridades de linguagem.

Sendo assim, a presente pesquisa tem objetivo descrever o resultado da validação aparente e de conteúdo da tecnologia assistiva "Amamentação em ação" junto a pessoas com deficiência visual de duas realidades, país Europeu e da América de Sul.

\section{MÉTODO}

Trata-se de um estudo de desenvolvimento e avaliação de tecnologia assistiva em saúde, cuja temática retrata a amamentação. Estudos deste tipo têm a finalidade de construir recursos e equipamentos que auxiliem na autonomia e independência de pessoas com deficiência melhorando sua qualidade de vida e promoção da saúde.

Neste estudo, o construto é a tecnologia assistiva, em formato de cordel, "Amamentação em Ação" que foi elaborada no Ceará - Brasil, apoiada em revisão de literatura e avaliação de especialistas na área, em seguida submetida à avaliação de pessoas cegas. Em Porto - Portugal o conteúdo recebeu adaptação por especialistas na área de amamentação para que expressões culturais fossem respeitadas, em seguida a tecnologia assistiva foi avaliada por cegos.

No Brasil, a coleta de dados ocorreu de março a maio de 2011, em Portugal de março a junho de 2012. A avaliação do construto foi realizada com acesso ao site www.labcomsaude.ufc.br, onde estavam disponíveis "Convite", "Termo de Consentimento Livre e Esclarecido", a tecnologia assistiva "Amamentação em Ação" e "Instrumento de Avaliação".

No Brasil, contou-se com apoio da Associação de Cegos do Estado do Ceará para a captação dos sujeitos do estudo. Em Porto, Portugal, os juízes especialistas e a aplicação da tecnologia com o público-alvo foi realizada na Escola Superior de Enfermagem do Porto (ESEP) e Associação de Cegos e Amblíopes de Portugal (ACAPO).

Pretendia-se inicialmente coletar dados com a população do estudo, as quais são Pessoas com Deficiência Visual associadas à ACAPO. Porém, pela inviabilidade de horários de alguns possíveis participantes e pesquisadores, a coleta dos dados foi realizada utilizando a técnica bola de neve.

A aplicação da tecnologia assistiva foi feita em computador, utilizando sintetizador de voz, sistema de leitura da tela do computador para cegos. Estes equipamentos, no Brasil, eram da associação onde foi realizada a pesquisa. Em Portugal, por sua vez, em muitos momentos, os equipamentos eram de uso pessoal da pesquisadora, pois eram escassos na Associação. Todos utilizados com sintetizador de voz, importante software que auxilia na leitura. Para as pessoas que não conseguiam acessar e manipular a tecnologia sozinha foram agendados encontros presenciais com a pesquisadora ou um mediador que pudesse supervisionar e auxiliar os sujeitos no acesso ao site. Para toda a coleta, a internet foi importante ferramenta. Esta, quando viável, permitiu aplicar a tecnologia de maneira efetiva e interferiu na avaliação de alguns itens relacionados a avaliação.

Instrumento de avaliação inqueria sobre perfil sociodemográfico; avaliação dos tópicos presentes denominados: objetivos, organização, motivação, design e navegabilidade da website; estrutura e apresentação da tecnologia. Para os itens de avaliação utilizou-se escala tipo Likert de um a cinco.

Para comparação das médias dos tópicos objetivos, organização, motivação, design e navegabilidade do website; estrutura e apresentação da tecnologia, as escalas foram transformadas de 0 a 100, por meio da expressão [(Valor tópico - Mín) / (Máx - Mín)]x100. Verificou-se a normalidade das variáveis por meio do teste de Kolmogorov-Smirnov. Comparação das características dos sujeitos entre os dois países foi realizada pelo teste de FisherFreeman-Halton e de Fisher. Médias entre os tópicos comparados pelo teste ANOVA e, se estatisticamente significante, comparações múltiplas realizadas pelo teste de Tukey. Médias dos tópicos entre os dois países comparadas pelo teste $\mathrm{t}$ de Student (se escala de 0 a 100) e teste de Mann-Whitney (se escala de 1 a 5). Consideraram-se como estatisticamente significantes, os resultados com $\mathrm{p}<0,05$.

Foram respeitados os aspectos éticos legais, cumprindo resolução 466/2012. Para realização do estudo em Porto-Portugal, foi considerada declaração de autorização, visto não existir Comitê de Ética em Pesquisa.

\section{RESULTADOS}

Participaram do estudo, 19 pessoas com deficiência visual do Brasil e 21 de Portugal. Na tabela 1 a seguir, consta o perfil destas pessoas. 
Tabela 1 - Caracterização dos sujeitos do Brasil e Portugal, 2012. $(n=19 / n=21)$

\begin{tabular}{|c|c|c|c|c|c|}
\hline \multirow{2}{*}{ Variável } & \multicolumn{2}{|c|}{ Brasil } & \multicolumn{2}{|c|}{ Portugal } & \multirow{2}{*}{$\mathbf{P}$} \\
\hline & $\mathrm{n}$ & $\%$ & $\mathbf{n}$ & $\%$ & \\
\hline Faixa Etária (ano) & & & & & $0,032^{*}$ \\
\hline $20-30$ & 9 & 47,3 & 6 & 28,6 & \\
\hline $31-40$ & 7 & 36,8 & 3 & 14,4 & \\
\hline $41-50$ & 3 & 15,9 & 4 & 19,0 & \\
\hline $51-60$ & - & - & 4 & 19,0 & \\
\hline$>60$ & - & - & 4 & 19,0 & \\
\hline Sexo & & & & & $0,525^{\dagger}$ \\
\hline Feminino & 9 & 47,4 & 13 & 61,9 & \\
\hline Masculino & 10 & 52,6 & 8 & 30,1 & \\
\hline Estado civil & & & & & $0,078^{(1)}$ \\
\hline Solteiro & 13 & 68,4 & 8 & 30,1 & \\
\hline Casado/União Estável & 6 & 31,6 & 8 & 30,1 & \\
\hline Viúvo & - & - & 4 & 19,0 & \\
\hline Outro & - & - & 1 & 4,8 & \\
\hline Anos de estudo & & & & & $0,045^{(1)}$ \\
\hline 4 & 1 & 5,3 & 6 & 30,0 & \\
\hline 9 & 15 & 78,8 & 8 & 40,0 & \\
\hline 12 & - & - & 1 & 5,0 & \\
\hline$>12$ & 3 & 15,9 & 5 & 25,0 & \\
\hline
\end{tabular}

${ }^{(1)} \mathrm{p}$ de Fisher-Freeman-Halton; ${ }^{\dagger} \mathrm{p}$ exato de Fisher

As variáveis que tiveram diferenças estatísticas significantes foram faixa etária $(p=0,032)$ e anos de estudo $(p=0,045)$. No Brasil, prevaleceram sujeitos jovens e com anos de estudo que correspondiam à conclusão do ensino fundamental. Em Portugal, houve distribuição homogênea entre as faixas etárias e, também, prevaleceram sujeitos com ensino fundamental. É importante ressaltar que ao contabilizar o número de participantes nesta variável tem-se vinte participantes, e isso refere-se a ausência de resposta de uma pessoa.

Sexo e estado civil não apresentaram diferenças estatísticas entre os dois países. No Brasil, o percentual de solteiros foi 2,2 vezes maior que o de casados. Em Portugal 19\% dos sujeitos eram viúvos.

Na tabela 2, consta a avaliação de cada item dos tópicos objetivo, organização e motivação pelos sujeitos da pesquisa.

Tabela 2 - Avaliação das médias de avaliação dos tópicos objetivos, organização e motivação. Brasil e Portugal, 2012.

\begin{tabular}{llll}
\hline \multicolumn{1}{c}{ Tópicos } & \multicolumn{1}{c}{ Brasil } & Portugal & \multirow{2}{*}{ p $^{*}$} \\
\cline { 2 - 3 } & \multicolumn{1}{c}{ Média; Med. } & Média; Med. & \\
\hline Objetivo (média \pm desvio padrão) & $97,3 \pm 4,9$ & $90,2 \pm 12,2$ & $0,019^{\dagger}$ \\
Aspectos da composição do leite materno & 4,$95 ; 5,0$ & 4,$86 ; 5,0$ & 0,630 \\
Esclarece dúvidas sobre mitos e tabus & 4,$89 ; 5,0$ & 4,$48 ; 5,0$ & 0,074 \\
Ressalta importância da família e profissional & 4,$89 ; 5,0$ & 4,$38 ; 4,0$ & 0,015 \\
Ressalta vantagens para criança & 5,$0 ; 5,0$ & 4,$95 ; 5,0$ & 0,810 \\
Ressalta vantagens para mãe & 5,$0 ; 5,0$ & 4,$81 ; 5,0$ & 0,307
\end{tabular}




\begin{tabular}{llll}
\hline \multicolumn{1}{c}{ Tópicos } & \multicolumn{1}{c}{ Brasil } & \multicolumn{1}{c}{ Portugal } & \multirow{2}{*}{ p $^{*}$} \\
\cline { 2 - 3 } & \multicolumn{1}{c}{ Média; Med. } & Média; Med. & \\
\hline Aborda comunicação em saúde & 4,$79 ; 5,0$ & 4,$33 ; 4,0$ & 0,065 \\
Retrata Incentivo à amamentação & 5,$0 ; 5,0$ & 4,$62 ; 5,0$ & 0,074 \\
Aborda mudança de comportamento e atitude & 4,$74 ; 5,0$ & 4,$62 ; 5,0$ & 0,436 \\
Atende objetivos das instituições que trabalham com & 4,$79 ; 5,0$ & 4,$43 ; 5,0$ & 0,124 \\
amamentação & & & \\
Organização (média \pm DP) & $91,0 \pm 11,2$ & $83,7 \pm 23,3$ & 0,225 \\
Tecnologia é atraente & 4,$47 ; 5,0$ & 4,$14 ; 5,0$ & 0,486 \\
Informações claras e termos compreensíveis & 4,$74 ; 5,0$ & 4,$38 ; 5,0$ & 0,307 \\
Tamanho da tecnologia está bom & 4,$11 ; 5,0$ & 4,$29 ; 5,0$ & 0,768 \\
Tópicos têm sequência lógica & 4,$79 ; 5,0$ & 4,$38 ; 5,0$ & 0,124 \\
Material está apropriado & 4,$74 ; 5,0$ & 4,$29 ; 5,0$ & 0,215 \\
Retrata aspectos chaves importantes & 5,$0 ; 5,0$ & 4,$62 ; 5,0$ & 0,124 \\
Motivação (média \pm DP) & $96,0 \pm 8,0$ & $84,1 \pm 24,2$ & 0,043 \\
Corresponde ao nível de conhecimento & 4,$0 ; 5,0$ & 4,$29 ; 5,0$ & 0,830 \\
Adequado para quem quer amamentar & 4,$95 ; 5,0$ & 4,$33 ; 5,0$ & 0,111 \\
Adequado para o pai incentivar aleitamento materno & 4,$79 ; 5,0$ & 4,$29 ; 5,0$ & 0,333 \\
Adequado para família incentivar aleitamento materno & 4,$79 ; 5,0$ & 4,$48 ; 5,0$ & 0,307 \\
\hline
\end{tabular}

"p de Mann-Whitney; ${ }^{\dagger}$ Teste $t$ de Student - Escala de 0 a 100; Med=Mediana; DP=desvio padrão; a Escala dos tópicos de 0 a 100; b Escala dos itens de 0 a 5

Nos dois países, todos os tópicos foram bem avaliados, com destaque para objetivo determinado pelos propósitos, metas ou finalidade a que se deseja com a utilização da tecnologia na modalidade de cordel. Comparativamente, o tópico Objetivo $(p=0,019)$ e Motivação $(p=0,043)$ foram diferentes estatisticamente. A média nos dois tópicos foi maior no Brasil.

Tópicos e itens tiveram excelentes avaliações com notas superiores a quatro. No tópico Objetivo, no item Ressalta importância da família e profissional, não houve diferença estatística $(p=0,015)$.
O Brasil obteve três itens com notas máximas, dos quais, vantagens da amamentação para a mãe, criança e incentivo para tal. Outro item com nota máxima está presente no tópico organização: retrata aspectos chaves importantes.

Em Portugal, todas as médias também foram excelentes. Com destaque para: aspectos da composição do leite materno e vantagens da amamentação para criança e mãe.

A tabela 3 descreve achados relacionados com os tópicos design, navegabilidade e, estrutura e apresentação, segundo os dois países.

Tabela 3 - Avaliação das médias dos tópicos design, navegabilidade e, estrutura e apresentação do web site. Brasil e Portugal, 2012.

\begin{tabular}{|c|c|c|c|}
\hline \multirow{2}{*}{ Tópicos } & Brasil & Portugal & \multirow{2}{*}{$\mathrm{p}^{*}$} \\
\hline & Média; Med. & Média; Med & \\
\hline Design (média \pm desvio padrão) & $97,3 \pm 7,8$ & 75,$0 ; 27,9$ & 0,002 \\
\hline Página inicial clara e pode ser manipulada & 4,$89 ; 5,0$ & 4,$0 ; 4,0$ & 0,002 \\
\hline Navegabilidade (média \pm DP) & $96,9 \pm 5,6$ & $87,7 \pm 24,6$ & 0,191 \\
\hline Facilidade entre páginas, seções e links & 4,$95 ; 5,0$ & 4,$41 ; 5,0$ & 0,066 \\
\hline Links de acesso às tecnologias estão definidos & 4,$79 ; 5,0$ & 4,$53 ; 5,0$ & 0,639 \\
\hline Links para acesso operam eficientemente & 4,$89 ; 5,0$ & 4,$59 ; 5,0$ & 0,490 \\
\hline
\end{tabular}




\begin{tabular}{|c|c|c|c|}
\hline \multirow{2}{*}{ Tópicos } & Brasil & Portugal & \multirow{2}{*}{$\mathrm{p}^{*}$} \\
\hline & Média; Med. & Média; Med & \\
\hline Estrutura e apresentação (média \pm DP) & $94,4 \pm 9,2$ & $81,8 \pm 23,7$ & 0,043 \\
\hline $\begin{array}{l}\text { Organização clara e lógica para localização da } \\
\text { tecnologia }\end{array}$ & 4,$89 ; 5,0$ & 4,$29 ; 5,0$ & 0,052 \\
\hline Disponibiliza informação para acesso à tecnologia & 4,$63 ; 5,0$ & 4,$35 ; 5,0$ & 0,315 \\
\hline Informações organizadas e facilmente entendidas & 4,$79 ; 5,0$ & 4,$38 ; 5,0$ & 0,205 \\
\hline Informações apresentadas nos links é apropriada & 4,$95 ; 5,0$ & 4,$35 ; 5,0$ & 0,030 \\
\hline Apresentação contribui para aprendizado & 4,$53 ; 5,0$ & 4,$10 ; 5,0$ & 0,215 \\
\hline Carta convite estimula acesso à tecnologia & 4,$63 ; 5,0$ & 4,$12 ; 4,0$ & 0,121 \\
\hline Apresentação do TCLE adequada & 4,$95 ; 5,0$ & 4,$43 ; 5,0$ & 0,124 \\
\hline Apresentação Instrumento de avaliação adequada & 4,$84 ; 5,0$ & 4,$62 ; 5,0$ & 0,611 \\
\hline
\end{tabular}

"p de Mann-Whitney; ${ }^{\dagger}$ Teste $t$ de Student - Escala de 0 a 100; DP = desvio padrão; Med = Mediana

Comparação entre Brasil e Portugal, evidenciou que a média do Brasil que foi superior à de Portugal refere-se a design $(\mathrm{p}=0,002)$. Este tópico é determinado pelo desenho apresentado na página, demonstrando clareza em sua manipulação. Estrutura e apresentação mostraram diferença estatisticamente significante quando comparadas as médias dos dois países $(\mathrm{p}=0,043)$, determinado pela forma de apresentar a tecnologia. Envolve organização geral, estrutura, estratégia de apresentação, coerência e suficiência. $\mathrm{O}$ item que apresentou diferença estatística foi sobre o conteúdo das informações apresentadas nos links ser apropriado. Item de melhor avaliação no Brasil.

Melhores avaliações no Brasil correspondem aos tópicos Design e navegabilidade. Em Portugal a melhor avaliação foi navegabilidade, determinada pela facilidade do usuário navegar nas páginas e conteúdos correspondentes. Os itens de melhor avaliação, por sua vez, constituintes dos tópicos navegabilidade e estrutura e apresentação, respectivamente: links para acesso operam eficientemente e apresentação instrumento de avaliação adequada.

\section{DISCUSSÃO}

No Brasil houve mais participantes jovens, dado que diferente do retrato nacional encontrado neste país, o qual traz que o número de pessoas com deficiência visual é maior em indivíduos acima de 65 anos. $^{3}$ Esta ocorrência pode ser atribuída ao fato da amostra não seguir critério probabilístico de seleção. Em Portugal, o maior número de sujeitos participantes reflete a realidade predominante. Há evidências de que a deficiência, de uma forma geral, afeta com frequência os sujeitos mais velhos. ${ }^{9}$
Em Portugal a população de idosos subiu 7,0\% em 2001 para 9,7\% em 2013. A tendência é mais acentuada entre as mulheres com 8,6 \% em 2001 e $11,5 \%$ em 2013. A população desta faixa etária representa quase metade da população idosa, 49,0\% em 2013, valor que sobe para $51,8 \%$ nas mulheres. A proporção dos mais idosos (80 ou mais anos), em 2013 , eleva-se a $27,9 \%$, sendo $23,8 \%$ nos homens e $30,8 \%$ nas mulheres, diferença que ressalta o fenômeno da sobre mortalidade masculina, contra $18,1 \%$ e $24,1 \%$, respectivamente para homens e mulheres, em $2001 .{ }^{10}$ Esta pesquisa também retrata a realidade encontrada concernente ao número de mulheres maior que homens, assim como na estatística ora apresentada.

Registro em Portugal aponta que a maioria da população idosa (55\%) que apresenta mobilidade reduzida ou não consegue andar, inclusive sequer conseguem realizar atividades relacionadas com a higiene pessoal (tomar banho/vestir-se), vive sozinha ou acompanhada por outros idosos. ${ }^{9}$

Em relação aos anos de estudo, tanto no Brasil como em Portugal, os sujeitos tiveram sua maior proporção em torno de nove anos de estudo, ou seja, até o ensino fundamental. Observa-se, portanto, que os indivíduos com deficiência em grande parte apresentam apenas poucos anos de estudo.

Ao longo de uma história de preconceitos e discriminação, muitos movimentos de exclusão foram gerados em vários níveis da sociedade. Isso acontece desde a antiguidade, em que mulheres, estrangeiros, deficientes e demais pessoas consideradas fora da normalidade pela sociedade eram excluídas, mas o fenômeno na época era tido como natural. Mais tarde, os efeitos dessa exclusão despontam, gerando desemprego prolongado onde 
muitos passam a ser socialmente excluídos. Nesta época, a exclusão passa a ser tema centralizador nos diversos meios da sociedade, e ainda refletiu na educação, dificultando e limitando o acesso às pessoas com deficiência. Inclusão na escola supõe considerações que ultrapassam a simples inovação educacional, e implicam o (re)conhecimento das peculiaridades dessa clientela. ${ }^{11}$

Em avaliação dos tópicos e itens, houve análise positiva da tecnologia assistiva pelas pessoas com deficiência visual tanto no Brasil como em Portugal, demonstrando que, embora existissem indivíduos que desconhecessem a tecnologia no formato de cordel, após leitura, a mesma foi considerada adequada em seus vários aspectos.

Com relação aos tópicos, apresentaram diferença estatística objetivo, motivação, design e, estrutura e apresentação. Isso mostra que a tecnologia assistiva apresenta assuntos essenciais e esclarecedores para quem quer amamentar e, além disso, para quem quer ajudar a amamentação, descrição clara e forma de apresentação convidativa ao acesso e curiosidade e, estrutura e apresentação lógica com informações necessárias para o acesso à tecnologia e instrumentos. Embora todas as avaliações tenham sido excelentes, no Brasil, as notas foram melhores, possivelmente estas avaliações podem estar relacionadas a cultura do cordel que, no Brasil, é mais presente, principalmente na região Nordeste, local onde foi realizada a pesquisa.

Especificamente no Brasil, as melhores avaliações foram dos tópicos objetivo e design. Em Portugal, por sua vez, os sujeitos também consideraram adequado o tópico objetivo, como melhor média, e, diferente do Brasil, navegabilidade teve uma das melhores notas. Esta pode está relacionado ao melhor acesso à internet.

Neste liame, percebe-se que o tópico objetivo obteve uma das melhores médias nos dois países, assim como seus itens. Este tópico retrata os propósitos a serem almejados com a utilização da tecnologia. Apresentou, no Brasil, três itens com avaliação máxima, dos quais: ressalta vantagens para a criança $(5,0)$, para a mãe $(5,0)$ e incentivo à amamentação $(5,0)$ e, em Portugal, também três itens com excelentes avaliações: aspectos da composição do leite materno $(4,86)$, vantagens da amamentação para a criança $(4,95)$ e para a mãe $(4,81)$.

Como amplamente difundido, o ato de amamentar proporciona diversos benefícios biológicos para o filho e é poderosa atividade de interação e relação amorosa entre mãe e bebê, em especial por favorecer a comunicação e formação de laços afe- tivos. É nesta atividade que a criança é abraçada, colocada junto ao corpo da mãe, acariciada. Ademais, constantemente, a mãe fala com ela. Embora aos cegos falte a visão, eles usam outros sentidos com maior precisão e necessitam de assistência à semelhança das pessoas que enxergam. Neste intuito, assim como o enfermeiro utiliza a criatividade e desenvolve tecnologias educativas para as demais pessoas, utilizar tecnologias assistivas para os cegos também é fundamental. ${ }^{12}$

Importante mencionar que neste mesmo tópico, houve forte concordância entre os dois grupos quanto ao item importância da família e do profissional da saúde para estímulo e a manutenção do aleitamento materno. A existência de pessoas presentes no cotidiano da lactante, principalmente mãe, avó, sogra e companheiro, interferem na prática da amamentação, influência maior quanto maior for o tempo de convivência. ${ }^{13}$

O enfermeiro deve apoiar e incentivar a lactante durante todo o ciclo gravídico puerperal para a prática do aleitamento materno. Além de informar sobre os benefícios fisiológicos, biológicos, psicológicos, emocionais e financeiros da amamentação para o binômio mãe-filho. ${ }^{14}$ Essas ações podem contribuir, ou não, para o início e manutenção da amamentação, a depender das atividades desenvolvidas pelos enfermeiros como o acolhimento, esclarecimento de dúvidas e orientações. Acredita-se que tecnologias educativas auto-instrucionais reforçem a abordagem do profissional de saúde.

Há o reconhecimento da importância do apoio do companheiro (família) para obtenção de melhores indicadores do aleitamento materno, dentre eles maior duração da amamentação. ${ }^{16}$ Também são determinantes para o abandono do aleitamento materno exclusivo aos lactantes mulheres que não recebiam auxílio do companheiro nos cuidados com a criança. ${ }^{17}$

Ainda com as melhores avaliações dos tópicos, no Brasil, foi design e em Portugal, navegabilidade. No tópico design, o item página inicial clara e pode ser manipulada obteve diferença estatística significante, embora com excelentes avaliações, uma das melhores notas apresentadas no Brasil. Concernente ao design, o mesmo retrata o desenho apresentado na página e, com isto, a facilidade de manipulação. Pela avaliação dos participantes, reforça que a página pode ser utilizada por pessoas com deficiência sem dificuldades, embora, no Brasil, a dificuldade seja menor. Isto também pode estar relacionado ao fato da possibilidade de acessar à internet em Portugal, que em alguns momentos, não foi efetivo. 
Literatura refere que descobertas sobre design apropriado à pessoa com deficiência visual são constantes. Discutem-se novas formas de produzir e disseminar conteúdos e produtos, diagnósticos e tratamentos, relações e informações acessíveis e possíveis às pessoas com deficiência visual, explorando todos os sentidos da percepção humana. ${ }^{18}$ Com a ajuda de novas tecnologias o design vem se apropriando de estrutura e aparência, no intuito de melhorar a comunicação. No caso de publicações direcionadas às pessoas com deficiência visual é preciso explorar os outros sentidos, para que esses supram a necessidade da visão. ${ }^{19}$

É visível a propagação de recursos elaborados para os deficientes visuais, dentre os quais: cardápios em Braille em restaurantes e lanchonetes, semáforos com sinal sonoro, pisos diferenciados nas calçadas. Porém, ainda existe precariedade nos serviços específicos e ausência de incentivo para melhorar a qualidade de vida desta clientela, situações que dificultam a sociedade ser inclusiva e priorizar o desenho universal. ${ }^{20}$

Quanto à navegabilidade, outra melhor nota em Portugal, é variável influenciada diretamente na utilização da internet. Isto significa que o público que acessava à tecnologia assistiva quando dispunha de internet, tinha boa conexão. Navegabilidade é ferramenta necessária à inclusão digital, entendido como acesso às tecnologias da informação, estratégias inclusivas que favorecem esse processo. Inclusão também está atrelada ao desenvolvimento de tecnologias assistivas que ampliem a acessibilidade e consequente autonomia e independência para pessoas com deficiência. $\mathrm{O}$ analfabetismo digital, ao afetar a capacidade de aprendizado, navegabilidade e divulgação de informações, gera consequências em todas as esferas da vida de um ser humano. ${ }^{21}$

Destaque é dado à necessidade de ampliação e desenvolvimento de programas que ofertem oportunidades para práticas letradas digitais. Aponta para a necessidade de políticas de planejamento e melhoria das estruturas informacionais das escolas públicas; investimento tanto em equipamentos para inclusão efetiva das tecnologias da informação e comunicação (TICs) no ensino, quanto na formação humana adequada para atender à demanda de promoção de (multi)letramento(s) digital(is); de ampla discussão acadêmica e política sobre a função das TICs no desenvolvimento social e tecnológico, bem como na conquista de cidadania digital plena. ${ }^{22}$

Vale lembrar que para avaliação dos tópicos relativos a design, navegabilidade, estrutura e apresentação, o cego precisava ter acesso à internet viável para ouvirem a tecnologia de maneira ideal a cada um. Para a pessoa com deficiência visual, o sintetizador de voz e a velocidade da internet são primordiais para permitir inclusão digital e acessibilidade. Neste sentido, o item informações apresentadas nos links foi considerada apropriada, presente no tópico estrutura e apresentação, teve diferença estatística, pois a utilização da internet no Brasil, no local do estudo, foi mais viável e assim possível utilizar a tecnologia disponível e ter o acesso necessário ao conteúdo sobre amamentação.

Neste sentido, é importante discutir elaboração, avaliação e utilização das tecnologias em saúde, nas relações entre profissionais e usuários do sistema de saúde. Na enfermagem, como nas demais áreas da saúde, deve-se usufruir das tecnologias para assistir a clientela e, promover autonomia e independência através da educação em saúde. ${ }^{23}$ Ao construir tecnologias assistivas é essencial submetê-las, também, à avaliação da clientela, considerando particularidades e características, apresentando metas a serem alcançadas, motivando o leitor a ler e/ou escutar a tecnologia, além de adotar linguagem clara e objetiva em todos os passos para acesso e avaliação, evitar termos técnicos, frases complexas, negativas e longas, que tornam a leitura confusa.

Construir tecnologia implica realizar validação, fase importante do desenvolvimento e adaptação de construtos, deve englobar medidas para avaliar validade e confiabilidade; procedimentos fundamentais para pesquisadores e profissionais da área de saúde envolvidos em aproveitar e empregar cada vez mais medidas e instrumentos confiáveis e apropriados para determinado público. ${ }^{24}$

Para validação, faz-se necessário que os avaliadores tenham expertise na área de interesse do construto, pois assim avaliarão adequadamente a representatividade e relevância do material submetido à análise; ressaltar que as observações e sugestões dos juízes contribuem para aprimorar o conteúdo das questões e o agrupamento de ações. ${ }^{25}$ Porém, o que de fato consolida a validação é o teste com o público-alvo.

\section{CONCLUSÃO}

A tecnologia retrata a composição do leite materno, com esclarecimentos e apontamentos significativos para quem quer amamentar. É atraente, com informações claras e termos compreensíveis; tem sequência lógica e aborda aspectos-chave importantes. Ademais, permite transferência e generalização do conteúdo em diferentes contextos. 
Como observado nesta pesquisa, captar sujeitos cegos é difícil e laborioso. Entretanto, os participantes avaliaram, positivamente, a tecnologia, ou seja, gostaram da tecnologia assistiva, mas para avaliar utilizando o sintetizador e preencher o instrumento, é preciso tempo, e mesmo assim, muitas vezes, torna-se complexo. Muitos em suas atividades cotidianas se recusam a responder. Isto pode estar relacionado, ainda, à ausência da inclusão digital. Neste contexto, foi importante a presença de um mediador ou até mesmo do próprio pesquisador para efetuar estas etapas de captação.

É importante mencionar que o número de participantes foi importante para iniciar etapa de validação, com esta avaliação inicial da tecnologia. Neste sentido, é etapa importante e precisa ser continuado com o intuito de ampliar e completar a validação. Neste estudo, avaliação foi realizada, considerada positiva e eficaz pelo público.

\section{REFERÊNCIAS}

1. Silva KL, Roseni RS, Clarissa TS, Maria EOS, Laís AMF. Desafios da política, da gestão e da assistência para a promoção da saúde no cotidiano dos serviços. Rev REME.2012 Abr-Jun; 16(2):178-87.

2. World Health Organization [página na Internet]. Visual impairment and blindness. 2014 ago [cited 2017 jul 11]. Available from: http://www.who.int/ mediacentre/factsheets/fs282/en/

3. Instituto Brasileiro de Geografia e Estatística (IBGE). Censo Demográfico 2010: Características da População e dos Domicílios: Resultados do universo. Brasil: IBGE; 2010.

4. Associação de Cegos e Ambliopes de Portugal. 2002. [atualizado 2016 Fev 15; acesso 2014 Mai 30]. Available from: http://www.anacom.pt/render. jsp?categoryId=36666.

5. Cezario KG, Abreu MSN, Pagliuca LMF. Alimentação complementar do lactente: adaptação e avaliação de tecnologia de apoio para pais cegos portugueses. Rev Enferm Referência. 2014 Nov-Dez; 4(3):37-44.

6. Bersch R. Assistiva - Tecnologia e Educação. Porto Alegre. Rio Grande do Sul, 2013. [cited 2014 Mar 16] Available from: http://www.assistiva.com.br/ Introducao_Tecnologia_Assistiva.pdf.

7. Oliveira PMP, Carvalho ALRF, Pagliuca LMF. Cultural adaptation of educative technology in health: string literature with a focus on breastfeeding. Texto Contexto Enferm. 2014 Jan-Mar; 23(1):134-141.

8. Pasquali L. Instrumentos psicológicos: fundamentos e práticas. Porto Alegre (RS): Editora Artmed; 2010.

9. Instituto Nacional de Estatística (INE). Saúde e incapacidades em Portugal 2011. Portugal, 2012 [cited 2017 Jul 12]. Available from: https:// www.ine.pt/ngt_server/attachfileu.jsp?look_ parentBoui $=149447974 \& a t t \_$display $=n \& a t t$ download $=y$

10. Anúario Estatístico de Portugal 2014. [cited 2015 May 16]. Available from: https://www.ine.pt/ngt_server/ attachfileu.jsp?look_parentBoui=249622090\&att_ display $=$ n\&att_download $=y$

11. Fumegalli RCA. Inclusão escolar: o desafio de uma educação para todos?[Internet]. Ijuí (RS): Universidade Regional do Noroeste do Estado do Rio Grande do Sul; 2012 [cited 2015 May 07]; Available from: http:/ / bibliodigital.unijui.edu.br:8080/xmlui/ bitstream/handle/123456789/716/ritamonografia. pdf? sequence $=1$

12. Pagliuca LMF, Barbosa GOL, Wanderley LD, Oliveira PMP. Análise da comunicação verbal e não-verbal de uma mãe cega e com limitação motora durante a amamentação. Rev Bras Enfermagem. 2011 Mai-Jun; 64(3): 431-7.

13. Monte GCSB, Leal LP, Pontes CM. Rede social de apoio à mulher na amamentação. Cogitare Enferm. 2013 Dez [cited 2016 Jan 22]; 18(1):148-55. Available from: http:/ / revistas.ufpr.br/cogitare/article/view/31321.

14. Azevedo ARR, Alves VH, Souza RMP, Rodrigues DP, Branco MBLR, Cruz AFN. O manejo clínico da amamentação: saberes dos enfermeiros. Esc Anna Nery. 2015 Jul-Set [cited 2017 Jul 11]; 19(3):439445. Available from: http://www.redalyc.org/ pdf/1277/127741627007.pdf.

15. Inoue M, Binns CW, Otsuka K, Jimba M, Matsubara M. Infant feeding practices and breastfeeding duration in Japan: a review. Int Breastfeed J. 2012 [cited 2014 Jan 17]; 7(1):15. Available from: http://www.ncbi.nlm. nih.gov/pmc/articles/PMC3514256/

16. Machado MC, Assis KF, Oliveira FCC, Ribeiro AQ, Araújo RMA, Cury AF, Priore SE, Franceschini SCC. Determinants of the exclusive breastfeeding abandonment: psychosocial factors. Rev Saúde Pública. 2014Ouc; 48(6): 985-94.

17. Trevisan J. Design editorial para deficientes visuais. [TCC Internet]. Curitiba (PR): Universidade Tecnológica Federal do Paraná; 2012[cited 2016 Fev 04]. Available from: http://repositorio.roca. utfpr.edu.br/jspui/bitstream/1/3006/1/CT CODEG_2012_1_19.pdf

18. Paes F. Sites com Interfaces gráficas acessíveis a deficientes visuais: um estudo de caso de usabilidade. 2010 [cited 2011 nov 14]. Available from: http:/ / www. fernandopaes.ppg.br/acessibilidade/Interfaces_ Acessiveis_Fernando_Paes.pdf

19. Vicentini F. A utilização da impressão da linguagem Braille nas embalagens de medicamento em papel cartão [Internet]. Curitiba (PR): Universidade Tecnológica Federal do Paraná;2011. [cited 2015 May 07]; Available from: http://maua.br/files/monografias/aplicacaode-grafia-braile-em-papel-cartao-para-embalagens-deprodutos-farmaceuticos.pdf 
20. Mapa da inclusão digital/coordenação Marcelo Neri. Rio de Janeiro: Faculdade Getúlio Vargas, CPS, 2012 [cited 2013 mar 09] Available from http:/ / www.cps. fgv.br/cps/bd/Mid2012/MID_FT_FGV_CPS_Neri_ TextoPrincipal_Fim_GRAFICA_fim.pdf.

21. Saito FS, Ribeiro PNS. (Multi)letramentos(s) digital(is) e teoria do posicionamento: análise das práticas discursivas de professoras que se relacionaram com as tecnologias da informação e comunicação no ensino público. Rev Brase Linguística Aplicada. 2013 JanMar; 13(1): 37-65.

22. Oliveira PMP, Pagliuca LMF. Assessment of an educational technology in the string literature about breastfeeding. Rev Esc Enferm USP. 2013 Fev [cited 2015 Jul 09]; 47(1):205-12. Available from: http://www. scielo.br/scielo.php?script=sci_arttext\&pid $=$ S008062342013000100026\&lng=en\&nrm=iso.
23. Alexandre NMC, Coluci MZO. Content validity in the development and adaptation processes of measurement instruments. Ciênc Saúde Coletiva. 2011 JanJul; 16(7):3061-8. [cited 2015 jul 21]. Available from: http:/ / www.scielo.br/scielo.php?script=sci_arttext\&pid=S141381232011000800006\&lng=e\&nrm=iso.

24. Joventino ES, Almeida PC, Oriá MOB. The Maternal Self-efficacy Scale for preventing early childhood diarrhea: validity and reliability. Public Health Nursing. 2013 Mar; 30(2):150-8. [cited 2015 Dez 09]. Available from: http://onlinelibrary.wiley.com/ doi/10.1111/j.1525-1446.2012.01042.x/full.

25. Marques CAV, Figueredo EM, Gutiérrez MGR. Validation of an instrument to identify actions for screening and detection of breast cancer. Acta Paul Enferm. 2015 Mar-Abr; 28(2):183-9. 